\title{
QUALIDADE DA ASSISTÊNCIA DE ENFERMAGEM NA TERAPIA INTRAVENOSA PERIFÉRICA: ANÁLISE POR INDICADORES
}

Angela Elisa Breda Rodrigues de Souza', João Lucas Campos de Oliveira² ${ }^{2}$ Denise Costa Dias ${ }^{3}$, Anair Lazzari Nicola ${ }^{3}$

'Enfermeira. Universidade Estadual do Oeste do Paraná. Cascavel-PR-Brasil.

${ }^{2}$ Enfermeiro. Mestrando em Enfermagem. Universidade Estadual de Maringá. Maringá-PR-Brasil.

${ }^{3}$ Enfermeira. Doutora em Enfermagem. Universidade Estadual do Oeste do Paraná. Cascavel-PR-Brasil.

RESUMO: Pesquisa de delineamento longitudinal com abordagem observacional e quantitativa que teve como objetivo analisar a qualidade da assistência de enfermagem na terapia intravenosa periférica, por meio dos indicadores incidência de flebite, qualidade da cobertura e validade dos cateteres intravenosos periféricos. Os dados foram coletados de outubro de 2012 a agosto de 2013, em três unidades de um hospital de ensino do Estado do Paraná-Brasil, utilizando formulário próprio. Foram avaliados 174 pacientes e 221 cateteres. Os resultados indicaram incidência de flebite entre $20 \%$ e 25,33\%; a identificação e a validade dos acessos venosos foram classificadas, em sua maioria, como seguras e adequadas segundo análise do Índice de Positividade que classifica a atividade assistencial. Conclui-se que os indicadores avaliados podem ser interpretados, em sua maioria, como favoráveis à qualidade assistencial.

DESCRITORES: Enfermagem; Qualidade da assistência à saúde; Flebite; Cateterismo periférico.

\section{NURSING CARE QUALITY IN PERIPHERAL INTRAVENOUS THERAPY: ANALYSIS BY INDICATORS}

\begin{abstract}
This Longitudinal study, with an observational and quantitative approach, aimed to analyze the quality of nursing care in peripheral intravenous therapy, through the indicators, incidence of phlebitis, quality of coverage, and validity of the peripheral intravenous catheters. Data were collected from October 2012 to August 2013, in three units of a teaching hospital in the state of Paraná, Brazil, using a form created for this purpose. A total of 174 patients and 221 catheters were evaluated. The results indicated an incidence of phlebitis between 20\% and $25.33 \%$; identification and the validity of the venous accesses were classified as safe and adequate in the majority of cases, according to an analysis of the Positivity Index that classifies medical work. It was concluded that the evaluated indicators could mostly be interpreted as favorable for care quality.
\end{abstract}

KEYWORDS: Nursing; Quality of health care; Phlebitis; Peripheral catheterization.

\section{CUALIDAD DE LA ASISTENCIA DE ENFERMERÍA EN LA TERAPIA INTRAVENOSA PERIFÉRICA: ANÁLISIS POR INDICADORES}

RESUMEN: Investigación de delineación longitudinal con abordaje observacional y cuantitativa que tuvo como objetivo analizar la cualidad de la asistencia de enfermería en la terapia intravenosa periférica, por medio de los indicadores incidencia de flebitis, cualidad de la cobertura y validez de los catéteres intravenosos periféricos. Los datos fueron recogidos de octubre de 2012 a agosto de 2013, en tres unidades de un hospital de enseñanza del Estado de Paraná, Brasil, utilizando formulario propio. Fueron evaluados 174 pacientes y 221 catéteres. Los resultados apuntan incidencia de flebitis entre $20 \%$ y $25,33 \%$; la identificación y la validez de los accesos venosos fueron clasificadas como seguras y adecuadas en su mayoría, de acuerdo con análisis de Índice de Positividad que clasifica la actividad asistencial. Se concluye que los indicadores evaluados pueden ser interpretados, en su mayoría, como favorables a la cualidad asistencial.

DESCRIPTORES: Enfermería; Cualidad de la asistencia a la salud; Flebitis; Cateterismo periférico.
Autor Correspondente:

João Lucas Campos de Oliveira

Universidade Estadual de Maringá

Rua Carlos Weiss, 39 - 87020-310 Maringá-PR-Brasil

E-mail: enfjoaolcampos@yahoo.com.br
Recebido: $22 / 03 / 2014$

Finalizado: 29/06/2014 


\section{INTRODUÇÃO}

A qualidade da assistência prestada nos serviços de saúde é um tema socialmente relevante nos dias atuais. As instituições de saúde, representadas por organizações prestadoras de serviços, absorveram os preceitos da qualidade de forma processual, já que estes originam, essencialmente, da administração dos serviços de ordem manufatureira ${ }^{(1)}$.

Apesar de, ainda, não existir definição unânime do que se entende por Qualidade na Saúde ${ }^{(2)}$, na evolução conceitual e prática deste fenômeno, essa é concebida e aceita mundialmente como um conjunto de cuidados eficazes, eficientes, acessíveis, aceitáveis, equitativos e seguros ${ }^{(3)}$.

Diante do entendimento de Qualidade na Saúde, é importante mencionar o papel da equipe de enfermagem, já que o contato direto e frequente com os usuários assistidos acarreta em interferência incisiva na obtenção, manutenção ou queda da qualidade da assistência e da segurança desses ${ }^{(4)}$. Dessa forma, tal como na área da saúde como um todo, para garantir a qualidade da assistência de enfermagem deve-se, necessariamente, incluir a satisfação dos usuários assistidos $^{(5)}$.

Em que pese a qualidade da assistência no contexto da enfermagem, especialmente em ambientes hospitalares, cabe mencionar a importância desta equipe profissional na implantação, manutenção e cuidados com Cateteres Intravenosos Periféricos (CIP), isso porque estas são ações frequentes no manejo da terapia intravenosa de pacientes ${ }^{(6)}$.

Levando em consideração que a grande maioria dos pacientes hospitalizados necessita de, pelo menos, um acesso venoso, é necessário que atividades assistenciais, incluindo as relacionadas à terapia venosa, sejam avaliadas e controladas, no sentido de se alavancar a qualidade do serviço prestado e auxiliar o (re)planejamento da assistência ${ }^{(7)}$. Neste sentido, é possível avaliar a qualidade da assistência de enfermagem na terapia intravenosa, por meio de indicadores ${ }^{(7-9)}$. A saber, os indicadores, que podem ser considerados como instrumentos de gestão(10), são unidades de medida de uma atividade que possibilitam, aos profissionais de saúde, monitorar e avaliar os eventos que acometem os usuários, os trabalhadores, e as organizações, apontando processos e resultados organizacionais, objetivando a excelência do cuidado ${ }^{(1,9)}$.

No contexto da avaliação da qualidade da assistência de enfermagem, relacionada à terapia intravenosa mediante indicadores, dá-se destaque para a incidência de flebites; esta consiste numa complicação inflamatória da parede de uma veia $^{(11)}$. Por sua vez, o indicador de qualidade incidência de flebites é considerado como um dos principais que avaliam, especificamente, a qualidade da assistência de enfermagem ${ }^{(9)}$. Outros importantes indicadores dizem respeito à identificação do tempo de permanência (validade) e identificação da cobertura do CIP, já que se relacionam, diretamente, com a segurança das atividades assistenciais as quais o paciente está exposto $^{(8,12)}$.

Cumpre mencionar que estudos que abordam a avaliação da qualidade da assistência de enfermagem na terapia intravenosa são deveras importantes, isso porque, os mesmos podem auxiliar na identificação mais precisa de fatores que fundamentam melhorias no processo de cuidar, por meio do (re)planejamento das ações de enfermagem do serviço, o que consequentemente poderá resultar no avanço da própria qualidade assistencial.

Frente ao exposto, este estudo teve como questão norteadora: Como se apresenta a qualidade da assistência de enfermagem na terapia intravenosa periférica em um hospital público de ensino? E, como objetivo, analisar a qualidade da assistência de enfermagem na terapia intravenosa periférica por meio dos indicadores incidência de flebites, qualidade da cobertura e validade dos Cateteres Intravenosos Periféricos em pacientes internados em um hospital de ensino.

\section{MÉTODO}

Trata-se de uma pesquisa de delineamento longitudinal com abordagem observacional e quantitativa. Foi desenvolvida em um hospital de ensino do interior do Estado do Paraná-Brasil, em três unidades de internação. O hospital conta com uma capacidade operacional de 195 leitos, todos conveniados ao Sistema Único de Saúde (SUS), e as unidades contam com 26, 28 e 13 leitos, respectivamente, e são destinadas à internação para o atendimento clínico e 
cirúrgico em ortopedia e neurologia, tratamento clínico e cirúrgico geral, cardiologia e cirurgia cardiovascular, identificadas na instituição como G3, F2 e F1 ${ }^{(13)}$. A escolha destas unidades foi motivada pela maior possibilidade de encontrar pacientes em uso de CIP, já que em outros setores para adultos, como unidade de terapia intensiva e pronto socorro, usualmente os pacientes fazem uso de cateteres com inserção central.

A população alvo foi composta por todos os pacientes internados nas unidades escolhidas, que apresentassem uma ou mais inserções de CIP. A amostra foi composta pelos pacientes que aceitaram participar do estudo e que atenderam aos critérios de elegibilidade: idade igual ou superior a 16 anos, desde que, na vigência de idade menor que 18 anos, o paciente estivesse acompanhado por um responsável; com orientação preservada no tempo e no espaço, segundo avaliação por meio de questionamentos da pesquisadora; e com CIP inserido na unidade de internação.

A coleta de dados foi realizada entre outubro de 2012 a agosto de 2013 nas unidades G3 e F2, e no período de outubro a dezembro de 2012 na F1. Esta etapa da pesquisa teve tempo reduzido na última unidade mencionada porque a mesma teve os serviços interrompidos no hospital.

Para a coleta de dados, utilizou-se um formulário ${ }^{(6)}$ com os seguintes dados: identificação do paciente, unidade de internação, leito, diagnóstico médico, sexo, idade, data e hora da inserção do CIP, e rubrica do profissional que inseriu o cateter. Os casos de flebite foram evidenciados de acordo com a presença de sinais clínicos apresentados na Escala Visual de Flebites $^{(14)}$. O instrumento foi avaliado, por meio de teste piloto, com duração de sete dias e se mostrou adequado.

A coleta foi procedida da seguinte forma: a cada dia, a mesma pesquisadora compareceu às três unidades e identificou quais os pacientes haviam sido puncionados com CIP e se preenchiam os requisitos de elegibilidade. Em caso positivo o instrumento era então aplicado, para cada um dos pacientes e o local de inserção do CIP foi observado.

Foi realizado o acompanhamento de todos os pacientes por até 96 horas após a retirada do CIP, dentre os que permaneceram na unidade, com o intuito de detectar flebite pós infusional, e também, alguma possível não conformidade em relação à validade do cateter, já que o padrão de troca estabelecido pela Comissão de Controle de Infecção Hospitalar da instituição era de 72 horas.

A coleta de dados foi realizada por uma única pesquisadora e, preferencialmente, no período da noite, entre $19 \mathrm{~h} 30 \mathrm{~min}$ às $21 \mathrm{~h} 30 \mathrm{~min}$. $\mathrm{O}$ período escolhido foi em virtude do menor número de profissionais e visitantes nas unidades o que permitia avaliação de cada inserção sem interrupções. Em todos os casos onde foram verificados sinais clínicos de flebite, em qualquer grau de evolução e durante a vigência do acesso venoso, a equipe de enfermagem foi comunicada.

Os dados coletados foram transportados para planilhas eletrônicas do Microsoft Office Excel 2010 e a análise se deu da seguinte forma:

1) Para a análise da Incidência de Flebite utilizou-se duas fórmulas de indicadores ${ }^{(14-15)}$ : Infusion nursing standards of practice da Infusion Nurses Society (INS) e Manual de indicadores de enfermagem - NAGEH. Os dois parâmetros foram escolhidos pela não unanimidade nesta forma de mensuração em âmbito nacional. O cálculo segundo a INS ${ }^{(14)}$ considera o número total de pacientes e o número de casos de flebite, já a fórmula do NAGEH considera os casos de flebite e o número total de $\mathrm{CIP}^{(15)}$.

2) Para a análise da identificação e da validade dos CIP utilizou-se as fórmulas dos indicadores de instrumento validado ${ }^{(12)}$ acrescido do ajustamento para análise da qualidade, segundo padrões estabelecidos no Índice de Positividade (IP) ${ }^{(16)}$, que classifica a atividade assistencial avaliada em desejável, adequada, segura, limítrofe ou sofrível. A assistência desejável é aquela que atinge o IP em 100\%; assistência adequada entre 90 a 99\%; assistência segura entre 80 a 89\%; assistência limítrofe entre 71 a $79 \%$; e assistência sofrível com índice de positividade inferior a $70 \%{ }^{(16)}$.

A realização deste estudo cumpriu com todas as exigências éticas estabelecidas na Resolução CNS n. 466/2012, e obteve o parecer favorável de número 014/2011 do Comitê de Ética em Pesquisa da Universidade Estadual do Oeste do Paraná.

Os pacientes que compuseram o estudo foram orientados quanto aos objetivos e participação voluntária, além da manutenção do sigilo sob sua identificação. Houve a assinatura, em duas vias, do Termo de Compromisso Livre e Esclarecido pelo próprio participante, ou seu responsável 
quando menor de 18 anos de idade.

\section{RESULTADOS}

Os pacientes que aceitaram participar do estudo e atenderam aos critérios de elegibilidade resultaram em 174 participantes. Destes, 42,53\% eram do sexo feminino e $57,47 \%$ do sexo masculino; a idade oscilou entre 16 e 92 anos, com mediana 42 (Desvio interquartil $=16$ ); a maior concentração $(31,03 \%)$ dos pacientes avaliados tinha idade entre 21 a 30 anos. Do total de 174 pacientes foi possível avaliar 221 CIP.

A Tabela 1 demonstra os resultados obtidos quanto ao indicador Incidência de Flebites, por unidade, calculado de duas maneiras.
O Quadro 1 sumariza os resultados obtidos quanto a análise do indicador relacionado à qualidade da identificação dos CIP, conforme o índice de positividade e adequação aos padrões de qualidade da assistência de enfermagem.

O Quadro 2 apresenta os resultados encontrados na análise do indicador relacionado à qualidade da validade dos CIP, conforme o índice de positividade e adequação aos padrões de qualidade da assistência de enfermagem.

Tabela 1 - Incidência de flebites por unidade de internação. Cascavel-PR,Brasil, 2013

\begin{tabular}{lccccc}
\hline Unidade & N. de Pacientes & N. de CIP & Casos de flebite & IF (NAGEH) (\%) & IF (INS) (\%) \\
\hline G3 & 75 & 94 & 19 & 25,33 & 20,21 \\
\hline F2 & 79 & 102 & 19 & 24,05 & 18,62 \\
\hline F1 & 20 & 25 & 4 & 20,00 & 16,00 \\
\hline Total & 174 & 221 & 42 & 24,13 & 19,00 \\
\hline
\end{tabular}

Legenda: CIP: Cateter intravenoso periférico; IF: Incidência de Flebites; NAGEH: Núcleo de Apoio à Gestão Hospitalar; INS: Infusion Nurses Society.

Quadro 1- Análise da qualidade da identificação da cobertura dos cateteres intravenosos periféricos instalados nos pacientes. Cascavel-PR,Brasil, 2013

\begin{tabular}{|l|c|c|c|c|c|}
\hline Unidade & $\begin{array}{c}\text { Número de } \\
\text { observações }\end{array}$ & $\begin{array}{c}\text { Identificação } \\
\text { completa }\end{array}$ & $\begin{array}{c}\text { Identificação } \\
\text { inadequada ou } \\
\text { ausente }\end{array}$ & IP (\%) & QA \\
\hline G3 & 94 & 84 & 10 & 89,36 & Segura \\
\hline F2 & 102 & 81 & 21 & 79,41 & Limítrofe \\
\hline F1 & 25 & 24 & 1 & 96,00 & Adequada \\
\hline Total & 221 & 189 & 32 & - & - \\
\hline
\end{tabular}

Legenda: IP: Índice de Positividade; QA: Qualidade da assistência de enfermagem.

Quadro 2 - Análise da validade dos cateteres intravenosos periféricos. Cascavel-PR,Brasil, 2013

\begin{tabular}{|l|c|c|c|c|c|}
\hline Unidade & $\begin{array}{c}\text { Número de } \\
\text { observações }\end{array}$ & $\begin{array}{c}\text { CIP } \text { sin situ até } \\
\mathbf{7 2 h}\end{array}$ & $\begin{array}{c}\text { CIPS in situ mais } \\
\text { de } \mathbf{7 2 h}\end{array}$ & IP (\%) & QA \\
\hline G3 & 94 & 86 & 8 & 91,48 & Adequada \\
\hline F2 & 102 & 90 & 12 & 88,23 & Segura \\
\hline F1 & 25 & 23 & 2 & 92,00 & Adequada \\
\hline Total & 221 & 199 & 22 & - & - \\
\hline
\end{tabular}

Legenda: CIP: Cateter intravenoso periférico; IP: Índice de Positividade; QA: Qualidade da assistência de enfermagem. 


\section{DISCUSSÃO}

A incidência de flebites encontrada neste estudo foi próxima ao encontrado nas estatísticas do Programa de Compromisso com a Qualidade Hospitalar $(\mathrm{CQH})$, que também se baseia na fórmula do NAGEH. Para o ano de 2013, em hospitais gerais com mais de 50 leitos e de financiamento público, seladas ou não no $\mathrm{CQH}$, a mediana da incidência de flebite foi 0,20 ou $20 \%{ }^{(17)}$. Ainda, as incidências de flebites, encontradas em cada unidade de internação desta pesquisa, foram inferiores ou compatíveis a outros estudos brasileiros que tinham como referência a incidência como mensuração do evento e para a análise o número de pacientes com CIP, que resultaram $18,3 \%^{(18)}$ e $24,7 \%{ }^{(19)}$.

Apesar da difícil precisão da interpretação dos resultados, possivelmente, os achados podem significar direção positiva à qualidade da assistência de enfermagem. Isso porque os valores estão próximos aos encontrados por um Programa CQH e compatíveis a outros estudos que usam a incidência de acordo com o número de pacientes puncionados ${ }^{(18-19)}$. Além disso, os resultados da incidência de flebite são inferiores a um estudo similar que utilizou fórmula internacionalmente reconhecida e que resultou na incidência de $25,8 \%{ }^{(6)}$.

É importante observar que para comparar taxas de flebite é necessário que se demande atenção especial para o tipo de delineamento metodológico dos estudos selecionados, analisando-se os referenciais utilizados para o cálculo. Isto porque esta taxa tem sido calculada de maneiras diversas e, quando não há homogeneidade nos estudos, a comparação entre os mesmos é dificultada, ou até mesmo impossibilitada. Diante disso, a produção científica nacional sobre o tema não é homogênea, pois, como mencionado, encontramse estudos que calculam a incidência com base no referencial do $\mathrm{NAGEH}^{(18-19)}$, e também com base na fórmula proposta pelo INS ${ }^{(6)}$.

Possivelmente, uma forma de solucionar a problemática em relação à ausência de padronização no cálculo da incidência de flebites, é a realização de estudos de validação do indicador. Assim, comparações entre resultados de pesquisas seriam mais viáveis, pois variações nas definições e propostas de cálculos dificultam a análise mais assertiva, bem como podem comprometer a validade do indicador, que possui relevância para o controle da qualidade da assistência de enfermagem.

Em que pese a ocorrência de flebites, mesmo que em frequência reduzida, é um evento preocupante, já que este pode causar dor persistente e outras complicações, como infecção de tecidos moles e até sepse ${ }^{(20)}$, o que irá refletir no tempo de assistência de enfermagem, sofrimento para o paciente e familiares, e impacto financeiro para o paciente, familiares e instituições hospitalares ${ }^{(6)}$.

Diante do exposto, é importante que a equipe de enfermagem esteja apta a reconhecer, precocemente, os sinais de flebite. Menciona-se que este diagnóstico cabe ao enfermeiro, ato não delegável a outro profissional da equipe de saúde, pois implica em prescrição e seleção de intervenções inerentes ao cuidado, mesmo na existência de protocolo específico na instituição ${ }^{(7)}$.

Os resultados provenientes da análise dos indicadores, relacionados à qualidade da identificação e à validade dos CIP avaliados, podem ser interpretados como favoráveis à qualidade da assistência. Apesar disso, nenhum dos indicadores avaliados obteve resultado com índice de positividade em $100 \%$, que classifica a assistência de enfermagem em padrão de qualidade desejável. Com relação à identificação da cobertura dos CIP, uma das unidades foi classificada como limítrofe devido a ausência, ou inadequação, dos dados de identificação, fato que merece atenção.

Estudo recente também avaliou a identificação e a validade de CIP em dois hospitais universitários públicos, locais com o mesmo escopo do hospital avaliado nesta pesquisa e que utilizou da mesma maneira o índice de positividade, obteve como resultado assistência de enfermagem com indicadores variando em sofrível e limítrofe ${ }^{(8)}$. Os autores mencionam que, apesar de ambas as instituições apresentarem índices de positividade que não colocam a assistência de enfermagem em parâmetros entendidos como seguros ou adequados, um dos hospitais realiza a mensuração periódica de indicadores de qualidade da assistência de enfermagem, inclusive os relacionados à terapia intravenosa, e esta mesma instituição obteve valores do índice de positividade mais elevados ${ }^{(8)}$. 
A avaliação da qualidade e a segurança do cuidado prestado só são possíveis a partir dos registros realizados em documentos e ou formulários específicos, e devem refletir a continuidade da assistência. Em relação aos CIP, a documentação deve incluir, entre outras características, a identificação, indicação da pessoa que realizou o cuidado, data e hora da realização da punção venosa ${ }^{(14)}$.

Em relaçãoà validade, sugere-sequea rotina detroca deCIP a cada 72 a 96 horas não seja necessária, mas sim de acordo com a evidência ${ }^{(21)}$, indicação corroborada por outro órgão, de abrangência internacional, a Infusion Nurses Society. Esta entidade sugere que o rodízio dos CIP seja realizado, quando clinicamente indicado, seguindo critérios: avaliação da condição do paciente, local do acesso venoso, integridade da pele e do vaso cateterizado, tempo de terapia necessário, integridade e permeabilidade do dispositivo, tipo de cobertura e uso de dispositivo de estabilização ${ }^{(14)}$.

Apesar das indicações anteriormente mencionadas, na instituição em estudo, a rotina de troca dos CIP é a cada 72 horas, por normativa da Comissão de Controle de Infecção Hospitalar, indicando que parte da amostra avaliada possui inconformidades em relação à validade dos CIP.

\section{CONCLUSÕES}

O estudo permitiu a análise de indicadores de qualidade da assistência de enfermagem na terapia intravenosa periférica em um hospital universitário público. Ainda que não se tenha parâmetros de qualidade quanto à incidência de flebites alusivos na literatura, este indicador, nas duas formas em que foi calculado e nas três unidades de internamento avaliadas, se mostrou compatível ao encontrado nas estatísticas de um programa de qualidade hospitalar brasileiro, e inferior a compatível com estudos nacionais que utilizaram a incidência para a mensuração do evento.

Os indicadores de qualidade referentes à identificação e à validade de cateteres venosos periféricos obtiveram resultados que podem ser interpretados, em sua maioria, como favoráveis à qualidade da assistência de enfermagem, já que os mesmos tiveram a assistência classificada em maior frequência como adequada e segura, e apenas uma unidade avaliada, em relação à identificação dos cateteres, obteve assistência limítrofe.
Levanta-se a hipótese de que os resultados poderiam ser ainda mais favoráveis à qualidade se o hospital aderisse à apuração periódica de indicadores de qualidade assistencial, no cotidiano gerencial da organização.

Considera-se uma limitação do estudo a inexistência de análise que poderia permitir inferências. Todavia, a pesquisa traz uma contribuição valorosa para a instituição estudada, e também, para formação de um arcabouço teórico cientificamente respaldado que direcione ações voltadas à qualidade da assistência da enfermagem na terapia intravenosa.

Espera-se com a realização deste estudo, que novas pesquisas sejam fomentadas, com diferentes abordagens metodológicas, como por exemplo, o estabelecimento da relação de causa e efeito entre a presença de flebite e a inadequação da identificação e validade de cateteres venosos, ou outros indicadores. Por fim, que a utilização de indicadores de qualidade da assistência de enfermagem seja um tema mais discutido nos estudos e também na prática gerencial cotidiana das organizações de saúde.

\section{AGRADECIMENTOS}

Ao Centro de Ciências Biológicas e da Saúde da Universidade Estadual do Oeste do Paraná, pelo apoio ao desenvolvimento desta pesquisa.

\section{REFERÊNCIAS}

1. Tronchin DMR, Melleiro MM, Takahashi RT. A qualidade e a avaliação dos serviços de saúde e de enfermagem. In: Kurcgant $\mathrm{P}$, (organizadora). Gerenciamento de enfermagem. $2^{\mathrm{a}}$ ed. Rio de Janeiro: Guanabara Koogan; 2010. p. 71-83.

2. Seraponi M. Avaliação da qualidade em saúde. Reflexões teórico-metodológicas para uma abordagem multidimensional. Rev. Crit. Cienc. Soc. 2009;(85):6582.

3. World Health Organization (WHO). Quality of care: a process for making strategic choices in health systems. Geneva; 2006 [acesso em 19 jan 2014]. Disponível: http://www.who.int/management/quality/assurance/ QualityCare_B.Def.pdf

4. Magalhães ANM, Dall'Agnol CM, Marck PB. Nursing workload and patient safety - a mixed method study with an ecological restorative approach. Rev. Latino- 
Am. Enfermagem. [Internet] 2013;21(n.esp) [acesso em 19 jan 2014]. Disponível: http://dx.doi.org/10.1590/ S0104-11692013000700019

5. Pena MM, Melleiro MM. Degree of satisfaction of users of a private hospital. Acta Paul. Enferm. [Internet] 2012;25(2) [acesso em 25 mai 2014] Disponível: http:// dx.doi.org/10.1590/S0103-21002012000200007

6. Magerote NP, Lima MHM, Silva JB, Correia MDL, Secoli SR. Associação entre flebite e retirada de cateteres intravenosos periféricos. Texto Contexto Enferm. [Internet] 2011;20(3) [acesso em 22 out 2013]. Disponível: http://dx.doi.org/10.1590/S010407072011000300009

7. Lopes PC, Vendramim P, Stramasso LV. Indicadores relacionados à flebite. In: Leão ER, (organizadora). Qualidade em saúde e indicadores como ferramenta de gestão. São Caetano do Sul: Yedis; 2008.

8. Murassaki ACY, Versa GLGS, Bellucci Junior JA, Meireles VC, Vituri DW, Matsuda LM. Avaliação de cuidados na terapia intravenosa: desafio para a qualidade na enfermagem. Esc. Anna Nery. [Internet] 2013;17(1) [acesso em 19 jan 2014]. Disponível: http:// dx.doi.org/10.1590/S1414-81452013000100002

9. Gabriel CS, Melo MRAC, Rocha FLR, Bernardes A, Miguelaci T, Silva MLP. Utilização de indicadores de desempenho em serviço de enfermagem de hospital público. Rev. Latino-Am. Enfermagem. [Internet] 2011;19(5) [acesso em 19 jan 2014] 19(5). Disponível: http://dx.doi.org/10.1590/S010411692011000500024

10. Matsuda LM, Meireles VC, Gomes FV, Saalfeld SMS, Molina RM. Instrumentos administrativos: percepção de enfermeiros de um hospital universitário. Cogitare enferm. [Internet] 2010;15(1) [acesso em 19 jan 2014] Disponível: http://ojs.c3sl.ufpr.br/ojs2/index.php/ cogitare/article/view/17182/11317

11. Royal Collage of Nursing. RCN. Standards for infusion therapy. 3th ed. Londres: RCN; 2010.

12. Vituri DW, Matsuda LM. Content valid ation of quality indicators for nursing care evaluation. Rev Esc Enferm USP. 2009;43(2):429-37.

13. Hospital Universitário do Oeste do Paraná (HUOP), 2014 [Internet] [acesso em 19 jan 2014]. Disponível: http://www.unioeste.br/huop/

14. Infusion Nurses Society. Infusion nursing Standards of practice. J. Infus. Nurs. [Internet] 2011;34 [acesso em 19 jan 2014] Disponível: http://www.vardhandboken. se/Dokument/INS_2011.pdf

15. Manual de indicadores de enfermagem NAGEH / Compromisso com Qualidade Hospitalar (CQH). $2^{\text {a }}$ ed. São Paulo : APM/CREMESP; 2012.
16. Haddad MCFL. Qualidade da assistência de enfermagem: processo de avaliação em hospital universitário público [tese]. Ribeirão Preto (SP): Universidade de São Paulo; 2004.

17. Compromisso com a Qualidade Hospitalar $(\mathrm{CQH})$. Relatório de Indicadores Hospitalares; 2013. [Internet] [acesso em 19 jan 2014] Disponível: http://www.cqh. org.br/portal/pag/area.php?p_narea $=98$

18. Ferreira LR, Pedreira MLG, Diccini S. Flebite no pré e pós-operatório de pacientes neurocirúrgicos. Acta Paul. Enferm. 2007;20(1):30-6.

19. Urbanetto JS, Rodrigues AB, Oliveira DJ, Dornelles FF, Filho JMR, Gustavo AS, et al. Prevalência de flebites em pacientes adultos com cateter venoso periférico. REUFSM. 2011;1(3):440-8.

20. Gohil S, Balasubramanian S. Chronic neuropathic pain after peripheral venous cannulation. Anaesthesia. 2012;67:1395-7.

21. O'Grady NP, Alexander M, Burns LA, Dellinger $P$, Garland J, Heard S. et al. Guidelines for the Prevention of Intravascular Catheter-Related Infections, 2011. [Internet] [acesso em 19 jan 2014]. Disponível: http://www.cdc.gov/hicpac/pdf/guidelines/bsiguidelines-2011.pdf 